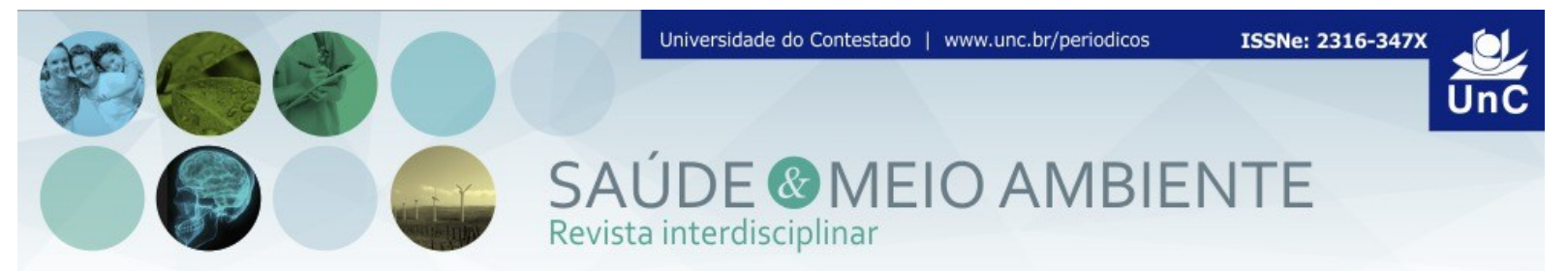

\title{
MONITORAMENTO DE FÁRMACOS NO RIBEIRÃO QUILOMBO, ESTADO DE SÃO PAULO, BR
}

Nilton Cesar Pasquini ${ }^{1}$

RESUMO: O monitoramento de fármacos residuais em rios e lagos vêm ganhando atenção dos pesquisadores e da população mundial. Este trabalho monitorou os fármacos antiinflamatório diclofenaco, ibuprofeno, naproxeno, paracetamol, piroxicam e os $\beta$-bloqueadores atenolol, metoprolol, nadolol, propranolol no Ribeirão Quilombo que abrange os municípios de Campinas, Hortolândia, Sumaré, Nova Odessa e Americana, estado de São Paulo-BR, desaguando no Rio Piracicaba no estado de São Paulo. A quantificação foi realizada por cromatografia gasosa (GCMS/MS), acoplado a um espectrômetro de massas. Avaliando os pontos podemos concluir que o ponto SU/NO apresentou as maiores concentrações. Pouco se sabe sobre o efeito do Atenolol, Metoprolol, Nadolol e Propranolol no meio ambiente aquático e seus efeitos ecotoxicológicos. Este estudo mostra que o Ribeirão Quilombo é castigado e consecutivamente leva este castigo para o Rio Piracicaba.

Palavras chave: Ribeirão Quilombo. Fármacos. Efluente. Meio ambiente aquático.

\section{DRUGS MONITORING IN RIBEIRÃO QUILOMBO, STATE OF SÃO PAULO, BR}

ABSTRACT: Monitoring of residual drugs in rivers and lakes have been gaining attention from researchers and the world's population. This study monitored the antiinflammatory drug diclofenac, ibuprofen, naproxen, paracetamol, piroxicane and $\beta$ blocker atenolol, metoprolol, nadolol, propranolol in Ribeirão Quilombo covering the municipalities of Campinas, Hortolandia, Sumaré, Nova Odessa and Americana, São Paul-BR, flowing into the Rio Piracicaba in São Paulo quantification was performed by gas chromatography (GC-MS / MS) coupled to a mass spectrometer. Assessing the points we can conclude that the SU / NO point showed the highest concentrations. Little is known about the effect of Atenolol, Metoprolol, Nadolol and Propranolol in the aquatic environment and their ecotoxicological effect. This study shows that the Ribeirão Quilombo is punished and consecutively takes this punishment to Rio Piracicaba.

Keywords: Ribeirão Quilombo. Drugs. Effluent. The aquatic environment.

\footnotetext{
${ }^{1}$ Mestrando em Química Tecnológica. Universidade Federal de São Carlos, São Carlos, São Paulo. Brasil. E-mail: nc.pasquini@ig.com.br
} 


\section{INTRODUÇÃO}

Para Tundisil (2003), embora dependam da água para sobrevivência e para o desenvolvimento econômico, as sociedades humanas poluem e degradam este recurso, tanto as águas superficiais quanto as subterrâneas. A diversificação dos usos múltiplos, o desejo de resíduos líquidos e sólidos em rios, lagos e represas relacionadas ao crescimento populacional e ao grau de urbanização têm produzido contínua e sistemática deterioração e perdas extremamente elevadas em quantidade e qualidade da água.

De acordo com Fent et al. (2006) concentrações de diferentes classes de fármacos como antiinflamatório, $\beta$-bloqueadores, regulados de lipídios, antibióticos etc, foram encontradas em diferentes países presentes em águas residuais, rios e córregos, oceanos, águas subterrâneas e até mesmo em água de consumo humano. Entre esses fármacos há o atenolol, metoprolol, nadolol, propranolol, diclofenaco, ibuprofeno, naproxeno, naracetamol e piroxican.

Goodman e Gilmam (2010) relatam que o fármaco atenolol foi descoberto em 1958 , e pertence à categoria dos $\beta$-bloqueadores ou também chamados $\beta$ adrenérgico, sendo um bloqueador seletivo de receptores $\beta 1$ - adrenérgicos. Ele é muito hidrofílico e parece penetrar no Sistema Nervoso Central (SNC) apenas em grau limitado. Essa categoria tem recebido enorme atenção clínica, devido à sua eficácia no tratamento da hipertensão, da cardiopatia isquêmica, da insuficiência cardíaca congestiva e de certas arritmias

Nadolol $\left[\mathrm{C}_{17} \mathrm{H}_{27} \mathrm{NO}_{4}\right]$ é indicado para hipertensão arterial, angina no peito, hipertireoidismo e enxaquecas. Após a absorção via oral, não é metabolizado pelo fígado e não sofre alteração ao ser excretado pela urina. O tempo de meia vida é de 12 a 24 horas (MCEVOY, 2003).

Segundo Mcevoy (2003), o metoprolol $\left(\mathrm{C}_{15} \mathrm{H}_{25} \mathrm{NO}_{3}\right)$ é indicado para tratamento pós infarto do miocárdio, diminui a pressão arterial. O composto é absorvido rapidamente pelo organismo e $95 \%$ é excretado pelos rins na forma de metabólitos como o alfa-hidroximetoprolol e cerca de $5 \%$ é eliminado sem alteração, o tempo de meia vida é de 3 a 7 horas.

$\mathrm{O}$ propranolol $\left[\mathrm{C}_{16} \mathrm{H}_{21} \mathrm{NO}_{2}\right.$ ] inibe a ação de agentes adrenérgicos e reduz a taxa de batimento e a força de contração muscular do coração, na redução da permeabilidade da membrana para os íons sódio, potássio e cálcio possuem ação anestésica loca (SWEETMAN, 2002).

O diclofenaco $\left(\mathrm{C}_{14} \mathrm{H}_{11} \mathrm{C}_{12} \mathrm{NO}_{2}\right)$ e o naproxeno $\left(\mathrm{C}_{14} \mathrm{H}_{14} \mathrm{O}_{3}\right)$ são anti-inflamatórios não esteroide (AINE), são um potente inibidor da enzima cicloxigenase é indicado para alivio de inflamação e dor.

O diclofenaco é derivado do ácido fenilacético, é administrado na forma de sal sódico ou potássio. As concentrações plasmáticas máximas são atingidas cerca de 30 minutos após a administração. Sua meia-vida terminal no plasma é de cerca de 
1-2 horas. No entanto, o diclofenaco é capaz de entrar no líquido sinovial onde as concentrações podem persistir e continuar a exercer uma resposta terapêutica, mesmo quando ocorre diminuição das concentrações plasmáticas (Fowler et al., 1983; Liaw et al., 1985). O diclofenaco é o AINE mais frequentemente prescrito em todo o mundo, classificado como o $8^{\circ}$ medicamento mais comercializado no mundo, tendo sido utilizado por mais de um bilhão de pacientes desde a primeira aprovação pelas autoridades sanitárias (GOTTIELB, 1985; GELLER et al., 2012).

O ibuprofeno $\left[\left(\mathrm{C}_{13} \mathrm{H}_{18} \mathrm{O}_{2}\right)\right]$ é um anti-inflamatório não esteroide (AINE), é indicado para alívio temporário da febre e dores moderada como dor de cabeça, cólica menstrual, artrite, dente e muscular. O paracetamol e o ibuprofeno, estão entre os fármacos mais consumidos no mundo (SENA et al., 2007). O ibuprofeno é uma droga não-esteroidal anti-inflamatória que também possui efeitos analgésico e antipirético. Algumas formulações farmacêuticas que combinam estes dois princípios ativos são usadas no tratamento de dores moderadas de origem reumática (HIGGGINS et al., 1999).

O paracetamol $\left[\left(\mathrm{C}_{8} \mathrm{H}_{9} \mathrm{NO}_{2}\right)\right]$, também conhecido como acetaminofeno, possui efeitos analgésico e antipirético similares aos da aspirina, apresentando a vantagem de não irritar a mucosa gastrointestinal (EL-ONEID et al., 1985). Não possui ação anti-iflamatória, age por inibição do ácido araquidônico impedindo a síntese das prostaglandinas, cerca de $90 \%$ de uma dose terapêutica são excretados na urina em 24 horas, sendo 1 a 4\% paracetamol inalterado e 20 a 30\% são metabólitos.

O piroxicam, [( $\left.\left.\mathrm{C}_{15} \mathrm{H}_{13} \mathrm{~N}_{3} \mathrm{O}_{4} \mathrm{~S}\right)\right]$ classificado como AINES derivado do oxicam, possui atividade anti-inflamatória, analgésica, antipirética e age inibindo as enzimas ciclo-oxigenases COX-1 e COX-2, de forma não seletiva. O piroxicam é prescrito no tratamento de varias doenças ginecológicas, tais como dismenorreia primária, endometriose, cisto hemorrágico, dor pós-operatória e não ginecológicas como a artrite reumatoide, osteoartrite, distúrbios musculoesqueléticos e gota.

\section{MATERIAL E MÉTODOS}

O estudo foi realizado nos meses de janeiro, março, maio, julho, setembro e novembro de 2014 em cinco pontos. Amostras de um litro de água superficial foram coletadas em cada ponto acondicionadas em vidro âmbar esterilizado e acondicionado em gelo. As análises foram realizadas em triplicatas na UFSCAR, Departamento de Química. 


\section{FÁRMACOS $\beta$-BLOQUEADORES}

Para determinar atenolol, metoprolol, nadolol, propranolol fez uso da metodologia de Osawa (2013), onde os padrões metoprolol (tartarato), Propranolol (cloridrato) e nadolol e o derivatizante BSTFA + 1\% TMCS utilizados, são padrões certificados da marca Sigma-Aldrich. Os solventes metanol, hexano, acetato de etila e acetona possuem grau UV/HPLC. As soluções padrões dos compostos foram preparadas em metanol e armazenadas a uma temperatura de $-4{ }^{\circ} \mathrm{C}$ numa concentração de $1000 \mathrm{~g} \mathrm{~mL}^{-1}$. Nas extrações em fase sólida (SPE) foram utilizados cartuchos $(6 \mathrm{~mL})$ contendo $1000 \mathrm{mg}$ de $\mathrm{C} 18$ da marca Chromabond ${ }^{\circledR}$. A separação foi realizada por cromatografia gasosa (GC-MS/MS) em uma coluna capilar de sílica HP-5msi (30m. 0,25 mm, 0,25 $\mu \mathrm{m})$, acoplado a um espectrômetro de massas, com temperatura de forno de $100{ }^{\circ} \mathrm{C}$ por minuto. A temperatura do injetor foi de $240{ }^{\circ} \mathrm{C}$, a do transfer line foi de $300{ }^{\circ} \mathrm{C}$ e a do íon source de $200{ }^{\circ} \mathrm{C}$. O gás hélio foi utilizado como gás de arraste com fluxo constante de $1 \mathrm{~mL}$ min. ${ }^{-1}$. Foram injetados $1 \mu \mathrm{L}$ de amostra em modo splitless.

\section{FÁRMACOS ANALGÉSICO E ANTI-INFLAMATÓRIO}

Para precisar a concentração de diclofenaco, ibuprofeno, naproxeno, paracetamol e piroxican fez uso de cromatografia líquida de alta eficiência (CLAE) adaptado da metodologia de Américo et al. (2012), onde as fases móveis constituíram-se de metanol (100\%) (fase A) e água Mili-Q (fases B), ambas acrescida de $0,1 \%$ de ácido trifluoracético (TFA). $O$ uso de um gradiente linear de $95 \%-50 \%$ de A por 15 minutos (a temperatura ambiente), com fluxo de1,0 ml min ${ }^{-1}$ foi utilizado na separação dos picos cromatográficos, pois alguns fármacos em fase móvel orgânica (acidificadas), decorrentes do aumento da concentração de principalmente os ácidos somente são eluídos metanol sobre a fase aquosa o que explica o uso da cromatografia em fase reversa (predominância da fase orgânica) para esse tipo de análise. De acordo com os diferentes pKas (constante de dissociação dos ácidos) para cada fármaco, inicialmente os compostos com menor tempo de retenção são os primeiros a eluírem e posteriormente os de maiores tempos de retenção. $O$ volume de injeção das amostras foi de $25,0 \mu \mathrm{L}$, sendo as amostras analisadas em triplicata. Os comprimentos de onda utilizados para a detecção dos picos cromatográficos foram de 240; 260 e $280 \mathrm{~nm}$. A identificação de cada fármaco foi efetuada de acordo com os seus respectivos tempos de retenção e também através de cada perfil espectrofotométrico. 


\section{ÁREA ESTUDADA}

A Bacia hidrográfica do Ribeirão Quilombo tem aproximadamente $396 \mathrm{~km}^{2}$ que abrange os municípios de Campinas, Hortolândia, Sumaré, Nova Odessa e Americana (PASQUINI, 2014a). O regime pluviométrico é tropical típico, com um período chuvoso, iniciando em outubro e terminando e abril, período de estiagem de maio a setembro, variando localmente o início de o término de cada um dos períodos, $85 \%$ da área é constituída de colinas amplas, com interflúvios superiores a $4 \mathrm{~km}^{2}$, topos extensos e aplainados, vertentes retilíneas a convexas, drenagem subdendrítica de baixa densidade, vales abertos e planícies aluniais restritas (PDRMNRQ, 2002; PASQUINI, 2012).

A calha natural do Ribeirão Quilombo, de su afluente principal, o Ribeirão Jacuba ou Hortolândia, e de outros afluentes sofreram as consequências da intensa conturbação parcial e em alguns casos total de suas bacias hidrográficas, podendose citar as seguintes: Córrego Pylles, do Parque e Galo em Americana; Córrego Capuava, em Nova Odessa; Pinheirimho e Tijuco Preto em Sumaré e Córrego Palmital entre Sumaré e Nova Odessa (PDMNRQ ; PASQUINI, 2014a).

A Figura 1, mostra a bacia do Ribeirão Quilombo, a nascente na cidade de Campinas e o desague no Rio Piracicaba após Americana.

Figura 1. Bacia do Ribeirão Quilombo.

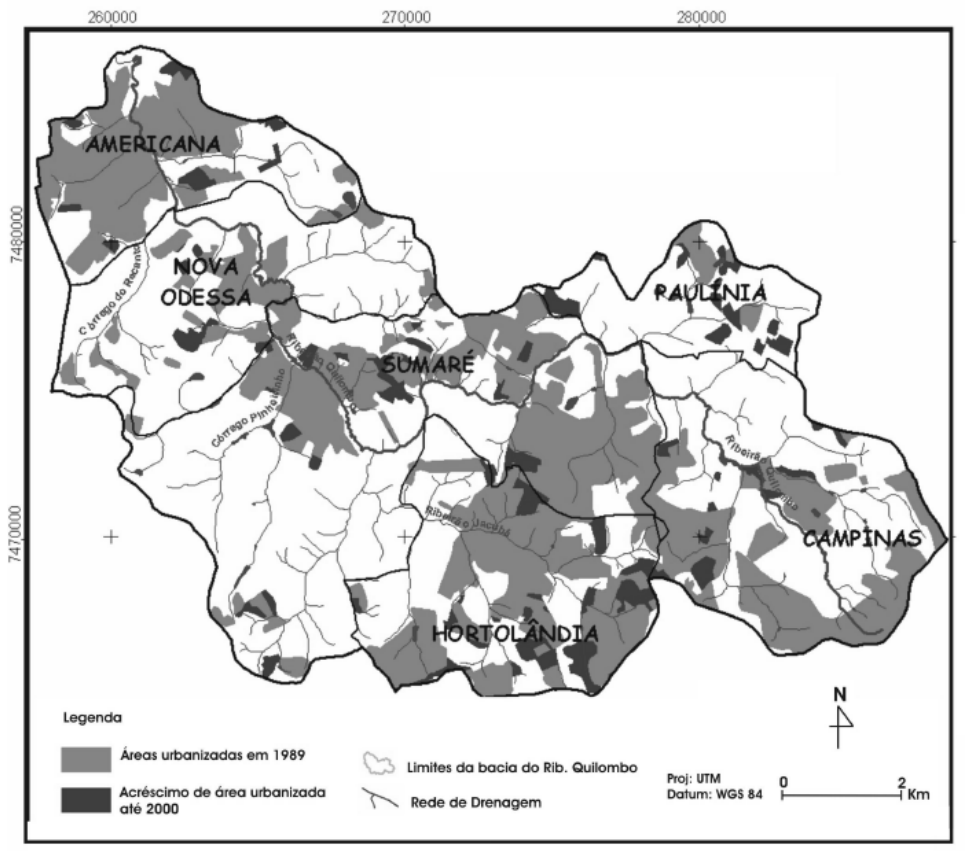

A Tabela 1 expõem a latitude e longitude dos pontos de coleta, onde CA/HO é entre os municípios de Campinas e Hortolândia, HO/SU entre Hortolândia e Sumaré, SU/NO entre Sumaré e Nova Odessa, NO/AM entre Nova Odessa e Americana e AM/RP entre Americana e o Rio Piracicaba. 
Tabela 1. Coordenadas geográficas dos pontos de coleta e a referência do município.

\begin{tabular}{c|c|c}
\hline Pontos de coleta & Latitude ( $\left.{ }^{\circ}\right)$ & Longitude ( $\left.{ }^{\circ}\right)$ \\
\hline CA/HO & $-22^{\circ} 82^{\prime} 4230^{\prime \prime}$ & $-47^{\circ} 14^{\prime} 0446^{\prime \prime}$ \\
\hline HO/SU & $-22^{\circ} 83^{\prime} 5903^{\prime \prime}$ & $-47^{\circ} 24^{\prime} 6747^{\prime \prime}$ \\
\hline SU/NO & $-22^{\circ} 80^{\prime} 3071^{\prime \prime}$ & $89^{\circ} 28^{\prime} 1079^{\prime \prime}$ \\
\hline NO/AM & $-22^{\circ} 75^{\prime} 8806^{\prime \prime}$ & $-47^{\circ} 31^{\prime} 5058^{\prime \prime}$ \\
\hline AM/RP & $-22^{\circ} 71^{\prime} 48883^{\prime \prime}$ & $-47^{\circ} 32^{\prime} 8088^{\prime \prime}$ \\
\hline
\end{tabular}

\section{RESULTADOS}

\section{FÁRMACOS $\beta$-BLOQUEADORES}

O atenolol foi encontrado em esgoto bruto na Itália $\left(0,49 \mathrm{ng} / \mathrm{L}^{-1}\right)$ e efluente de ETE $\left(0,28 \mathrm{ng} / \mathrm{L}^{-1}\right)$ por Castiglioni et al. (2006), Calamari et al. (2003) encontraram $0,050 \mathrm{ng} / \mathrm{L}^{-1} \mathrm{em}$ água superficial na Itália e Bendz e Paxéus (2005) determinaram no esgoto bruto da Suécia $\left(0,30 \mathrm{ng} / \mathrm{L}^{-1}\right)$ e $0,16 \mathrm{ng} / \mathrm{L}^{-1}$ no efluente de ETE. Na Tabela, observa-se que o ponto que mais apresentou o fármaco atenolol foi entre a cidade de Sumaré e Nova Odessa (SU/NO), sendo que o mês de março $\left(97,4 \mathrm{ng} / \mathrm{L}^{-1}\right)$ apresentou o maior índice, seguida pelo ponto NO/AM $\left(91,5 \mathrm{ng} / \mathrm{L}^{-1}\right)$ e AM/FIM $(90,5$ $\left.\mathrm{ng} / \mathrm{L}^{-1}\right)$, sendo ambos referentes ao mês de março. $\mathrm{Em}$ todos os meses o ponto SU/NO apresentou os maiores índices de fármacos, sendo $89,1 \mathrm{ng} / \mathrm{L}^{-1}, 97,4 \mathrm{ng} / \mathrm{L}^{-1}$, $54,6 \mathrm{ng} / \mathrm{L}^{-1}, 49,3 \mathrm{ng} / \mathrm{L}^{-1}, 61,7 \mathrm{ng} / \mathrm{L}^{-1}$ e $49,3 \mathrm{ng} / \mathrm{L}^{-1}$. O atenolol é consumido pela população na forma pura como associado com diuréticos como clortalidona ou hidoclortiazida $\left(\mathrm{C}_{7} \mathrm{H}_{8} \mathrm{~N}_{3} \mathrm{ClO}_{4} \mathrm{~S}_{2}\right)$, também com bloqueadores dos canais de cálcio como a anlopino $\left(\mathrm{C}_{20} \mathrm{H}_{25} \mathrm{ClN}_{2} \mathrm{O}_{5}\right)$, que é usado na medicina como vasodilatador coronário e hipotensor.

Tabela 2 - Concentração de Atenolol nos pontos analisados; nd significa que nada foi determinado.

\begin{tabular}{c|c|c|c|c|c|c}
\hline $\begin{array}{c}\text { Atenolol } \\
\text { ng/L }\end{array}$ & Janeiro & Março & Abril & Junho & Setembro & Novembro \\
\hline CA/HO & 9,6 & nd & 11,4 & 12,6 & 9,5 & 5,4 \\
\hline HO/SU & nd & 16,1 & nd & nd & nd & nd \\
\hline SU/NO & 89,1 & 97,4 & 54,6 & 49,3 & 61,7 & 49,3 \\
\hline NO/AM & 65,7 & 91,5 & 26,5 & 41,8 & 54,0 & 41,8 \\
\hline AM/FIM & 61,4 & 90,5 & 25,1 & 39,9 & 33,7 & 39,9 \\
\hline
\end{tabular}

$\mathrm{Na}$ Tabela 3 observa-se que a maior concentração de metoprolol foi observado entre os municípios de Sumaré e Nova Odessa (SU/NO) $\left(1601,5 \mathrm{ng} / \mathrm{L}^{-1}\right)$ no mês de janeiro.

Wiegel et al. (2004) encontrou $224 \mathrm{ng} / \mathrm{L}$ em rio da Suécia, Vieno et al. (2007) $107 \mathrm{ng} / \mathrm{L}^{-1}$ na Finlândia, Oswa encontrou $4658,2 \mathrm{ng} / \mathrm{L}^{-1}$ no Rio Atuba (PR) e 65,3 $\mathrm{ng} / \mathrm{L}^{-1}$ no Rio Iguaçu. 
Este fármaco é consumido de forma individual, apenas com este princípio ativo ou associado com anlopino $\left(\mathrm{C}_{20} \mathrm{H}_{25} \mathrm{CIN}_{2} \mathrm{O}_{5}\right)$ ou isoproterenol $\left(\mathrm{C}_{11} \mathrm{H}_{17} \mathrm{NO}_{3}\right)$.

Tabela 3 - Concentração de metoprolol nos pontos analisados; nd significa que nada foi determinado.

\begin{tabular}{c|c|c|c|c|c|c}
\hline $\begin{array}{c}\text { Metoprolol } \\
\text { ng/L }^{-1}\end{array}$ & Janeiro & Março & Abril & Junho & Setembro & Novembro \\
\hline CA/HO & 10,0 & 10,6 & nd & 34,6 & 146,7 & 164,2 \\
\hline HO/SU & 10,25 & nd & 21,6 & 31,1 & 86,4 & 98,7 \\
\hline SU/NO & 1654,5 & 1170,1 & 965,4 & 1051,6 & 865,4 & 975,0 \\
\hline NO/AM & 1601,5 & 801,5 & 947,0 & 653,4 & 801,6 & 897,4 \\
\hline AM/FIM & 639,4 & 465,1 & 256,1 & 156,7 & 234,9 & 167,5 \\
\hline
\end{tabular}

O fármaco nadolol apresentou maior concentração em março entre Sumaré e Nova Odessa (SU/NO) $\left(135,6 \mathrm{ng} / \mathrm{L}^{-1}\right)$ seguida do ponto entre Nova Odessa e Americana (NO/AM) do mesmo mês. Oswa (2013) no Rio Atuba determinou 123,8 $\mathrm{ng} / \mathrm{L}^{-1}$.

Tabela 4 - Concentração de nadolol nos pontos analisados; nd significa que nada foi determinado.

\begin{tabular}{c|c|c|c|c|c|c}
\hline $\begin{array}{c}\text { Nadolol } \\
\text { ng/L }\end{array}$ & Janeiro & Março & Abril & Junho & Setembro & Novembro \\
\hline CA/HO & nd & 5,6 & 10,3 & 11,3 & 5,1 & 7,1 \\
\hline HO/SU & nd & 4,5 & 8,6 & 10,5 & 6,4 & 8,1 \\
\hline SU/NO & 98,6 & 135,6 & 98,4 & 54,6 & 78,9 & 101,2 \\
\hline NO/AM & 101,2 & 126,5 & 111,1 & 53,6 & 86,5 & 100,9 \\
\hline AM/FIM & 85,6 & 101,3 & 65,5 & 36,4 & 79,4 & 91,4 \\
\hline
\end{tabular}

A maior concentração de propranolol foi no ponto SU/NO $\left(658,6 \mathrm{ng} / \mathrm{L}^{-1}\right) \mathrm{de}$ novembro seguido do mesmo mês um ponto depois NO/AM $\left(568,5 \mathrm{ng} / \mathrm{L}^{-1}\right)$; janeiro ponto SU/NO (551,6 ng/ $\left.\mathrm{L}^{-1}\right)$. Em estudos de monitoramento de efluentes de estações de tratamento de esgoto no Reino Unido, propranolol sempre foi encontrado na concentração média de $76 \mu \mathrm{g} / \mathrm{L}^{-1}$ (THOMAS; HILTON, 2004; WEIGEL et al., 2004), no Rio Atuba (PR) Oswa (2013) encontrou 946,1 ng/ $\mathrm{L}^{-1}$. Este fármaco é também encontrado associado com nortriptilina, um antidepressivo.

Tabela 5 - Concentração de propranolol nos pontos analisados; nd significa que nada foi determinado.

\begin{tabular}{c|c|c|c|c|c|c}
\hline $\begin{array}{c}\text { Propranolol } \\
\text { ng/L }\end{array}$ & Janeiro & Março & Abril & Junho & Setembro & Novembro \\
\hline CA/HO & 156,7 & 111,5 & 97,6 & nd & 27,6 & 27,1 \\
\hline HO/SU & 149,5 & 64,5 & 11,4 & 5,7 & 29,8 & 31,4 \\
\hline SU/NO & 551,6 & 271,5 & 368,5 & 327,6 & 435,9 & 658,6 \\
\hline NO/AM & 359,6 & 179,1 & 276,5 & 354,2 & 339,1 & 568,5 \\
\hline AM/FIM & 146,3 & 61,0 & 225,3 & 214,6 & 231,2 & 338,1 \\
\hline
\end{tabular}


Pesquisadores como Alder et al. (2010), Nolder et al. (2010), Varga et al. (2011), monitorando efluentes de estação de tratamento de esgotos (ETE) Hernando et al. (2007), Nolder et al. (2010) encontraram beta bloqueadores em águas superficiais.

O modo de ação dos fármacos no meio ambiente não é conhecido e muitas vezes não comente um, mas diferentes modos de ação podem ocorrer sobre esses organismos. A toxicidade, sobre os organismos, esta relacionada ao modo de exposição, aguda ou crônica (FENT et al., 2006). Deste modo, a presença de fármacos no meio ambiente pode gerar efeitos sutis sobre as espécies aquáticas e ainda resultar em risco à saúde humana pelo consumo de águas contaminadas (DORNE et al., 2007).

A ecotoxidade de alguns $\beta$-bloqueadores foi avaliada usando-se diferentes bioindicadores, demonstrando-se que o Propranolol é o mais tóxico, seguido de metoprolol e atenolol. A toxicidade do $\beta$-bloqueadores não foi ainda extensivamente estudada, com exceção do Propranolol que mostrou alta toxicidade aguda quando comparado a outros $\beta$-bloqueadores. O Propranolol também exibe toxicidade crônica, não somente sobre o sistema cardiovascular, mas também sobre o sistema reprodutivo de peixes (HUGGETT et al., 2002).

Outro interessante aspecto destes $\beta$-bloqueadores, diz respeito ao tratamento de efluentes, pois essas substâncias podem se comportar como surfactantes em pH neutro (MOSQUERA et al., 1999). Assim, é especulado que interações surfactantes presentes no meio ambiente podem afetar significativamente o comportamento dessa classe de fármacos, pois é sabido que surfactantes aniônicos possuem profunda influência sobre as propriedades das soluções (UPADHYAYA et al., 2006).

\section{FÁRMACOS ANALGÉSICO E ANTI-INFLAMATÓRIO}

Na Tabela 6 observa as concentrações encontradas para diclofenaco, sendo a maior concentração no ponto SU/NO $\left(2785,2 \mathrm{ng} / \mathrm{L}^{-1}\right)$, em todos os meses a maior concentração de diclofenaco foi no ponto SU/NO. Para diclofenaco, Castro et al. encontrou $2870 \mathrm{ng} / \mathrm{L}^{-1}$, na ETE de São Carlos-SP.

Américo et al. (2012) na ETE de Três Lagoas deparou com diclofenaco (2470 $\left.\mathrm{ng} / \mathrm{L}^{-1}\right)$, ibuprofeno $\left(2300 \mathrm{ng} / \mathrm{L}^{-1}\right)$, naproxeno $\left(4600 \mathrm{ng} / \mathrm{L}^{-1}\right)$, paracetamol $\left(130 \mathrm{ng} / \mathrm{L}^{-1}\right) \mathrm{e}$ piroxicam $\left(600 \mathrm{ng} / \mathrm{L}^{-1}\right)$. Kramer et al. (2012) precisou no rio Belém $729 \mathrm{ng} / \mathrm{L}^{-1}$ de ibuprofeno e $369 \mathrm{ng} / \mathrm{L}^{-1}$ de paracetamol, o diclofenaco foi analisado do rio Iguaçu e foi de $317 \mathrm{ng} / \mathrm{L}^{-1}$.

Ensaios laboratoriais realizados com truta arco-iris (Oncorhynchus mykiss) exposta a diferentes concentrações de diclofenaco relataram o acúmulo deste composto nos músculos, brânquias, rins e fígado dos animais (SCHWAIGER et al., 2004). Análises com medaka japonês (Oryzias latipes) revelaram que o diclofenaco 
possui potencial para causar toxicidade celular e agir como estrógenos nestes organismos (HONG et al., 2007).

Tabela 6 - Concentração de diclofenaco nos pontos analisados; nd significa que nada foi determinado.

\begin{tabular}{c|c|c|c|c|c|c}
\hline $\begin{array}{c}\text { Diclofenaco } \\
\text { ng/L }\end{array}$ & Janeiro & Março & Abril & Junho & Setembro & Novembro \\
\hline CA/HO & nd & 31,2 & 94,2 & 87,3 & 49,6 & 77,4 \\
\hline HO/SU & 22,1 & 30,0 & 137,2 & 129,4 & 90,5 & 39,5 \\
\hline SU/NO & 1845,5 & 2785,2 & 1902,5 & 1595,3 & 1601,6 & 1208,4 \\
\hline NO/AM & 1687,2 & 1984,2 & 1559,2 & 1208,9 & 1599,4 & 1298,7 \\
\hline AM/FIM & 1011,7 & 1193,9 & 1222,9 & 985,4 & 1398,5 & 947,3 \\
\hline
\end{tabular}

A Tabela 7, refere-se ao ibuprofeno, onde as maiores $\left(1184,3 \mathrm{ng} / \mathrm{L}^{-1}\right)$ concentrações foram observadas no ponto SU/NO, exceto no mês de novembro $\left(985,3 \mathrm{ng} / \mathrm{L}^{-1}\right)$.

Petrovic (2005) aludi que em análises realizadas no Rio Ebro os fármacos ibuprofeno e atenolol exibiram as seguintes concentrações $60 \mathrm{ng} / \mathrm{L}^{-1}$ e $72 \mathrm{ng} / \mathrm{L}^{-1}$.

Tabela 7 - Concentração de ibuprofeno nos pontos analisados; nd significa que nada foi determinado.

\begin{tabular}{c|c|c|c|c|c|c}
\hline $\begin{array}{c}\text { Ibuprofeno } \\
\text { ng/L }\end{array}$ & Janeiro & Março & Abril & Junho & Setembro & Novembro \\
\hline CA/HO & 13,5 & nd & 11,4 & 5,76 & 22,5 & 5,6 \\
\hline HO/SU & 7,5 & 5,6 & 9,4 & 5,03 & 29,4 & 8,5 \\
\hline SU/NO & 1033,4 & 129,5 & 884,2 & 129,4 & 1184,3 & 985,3 \\
\hline NO/AM & 1023,5 & 134,6 & 803,5 & 195,4 & 1044,2 & 994,3 \\
\hline AM/FIM & 985,4 & 131,0 & 799,4 & 201,4 & 1011,3 & 999,4 \\
\hline
\end{tabular}

O estudo toxicológico de Richards et al. (2004) a respeito do ibuprofeno apontou que este medicamento, em misturas com outros pode causar a morte de peixes, um aumento na abundância de fitoplâncton e zooplâncton, porém com uma diminuição da diversidade. Estudo com o crustáceo Daphnia magna revela que apenas concentrações acima de $10 \mathrm{mg} / \mathrm{L}^{-1}$ de ibuprofeno afetam significativamente as suas populações, causando então efeitos no crescimento, na reprodução e até inibição do organismo (HECKMANN et al., 2007). Pomati et al. (2004), revelaram que o ibuprofeno estimula o crescimento da cianobactéria Synechocystis $\mathrm{sp}$. Em uma concentração de $10 \mu \mathrm{g} . \mathrm{L}-1$ do fármaco e inibe o crescimento da macrófita Lemna gibba a $1 \mathrm{mg} / \mathrm{L}^{-1}$.

O naproxeno (Tabela 8 ) foram $554,8 \mathrm{ng} / \mathrm{L}^{-1}$ (SU/NO), $483,5 \mathrm{ng} / \mathrm{L}^{-1}$ (SU/NO), $466,7 \mathrm{ng} / \mathrm{L}^{-1}$ (NO/AM) e $399,4 \mathrm{ng} / \mathrm{L}^{-1}$ (SU/NO). Bioensaios com naproxeno e alguns de seus subprodutos da fotodegradação indicaram que o composto afeta a mobilidade e reprodução de crustáceos, o crescimento de algas e a reprodução de rotíferos podendo levar a morte destes organismos (ISIDORI et al., 2005). 
Tabela 8 - Concentração de naproxeno nos pontos analisados; nd significa que nada foi determinado.

\begin{tabular}{c|c|c|c|c|c|c}
\hline $\begin{array}{c}\text { Naproxeno } \\
\text { ng/L }\end{array}$ & Janeiro & Março & Abril & Junho & Setembro & Novembro \\
\hline CA/HO & 23,5 & nd & 23,5 & nd & 7,2 & 11,4 \\
\hline HO/SU & 26,3 & 7,49 & 33,3 & 28,3 & 43,5 & 28,4 \\
\hline SU/NO & 174,3 & 85,3 & 554,8 & 299,9 & 483,5 & 399,4 \\
\hline NO/AM & 172,0 & 77,4 & 155,4 & 291,7 & 466,7 & 389,2 \\
\hline AM/FIM & 169,8 & 71,3 & 111,1 & 287,0 & 341,1 & 345,2 \\
\hline
\end{tabular}

O paracetamol (Tabela 9) apresentou a maior concentração no ponto SU/NO $\left(305,1 \mathrm{ng} / \mathrm{L}^{-1}\right)$ no mês de novembro seguida por SU/NO $\left(284,5 \mathrm{ng} / \mathrm{L}^{-1}\right)$ no mês de junho, NO/AM $\left(183,1 \mathrm{ng} / \mathrm{L}^{-1}\right)$ em junho, NO/AM $\left(184,3 \mathrm{ng} / \mathrm{L}^{-1}\right)$ e SU/NO $\left(172,1 \mathrm{ng} / \mathrm{L}^{-1}\right)$ em setembro. Kim et al. (2007) afirma que o paracetamol apresenta um alto risco de toxicidade em ambientes aquáticos e afirma também que ainda é necessário mais estudo para saber o real impacto deste fármaco. Estudo demonstrou um aumento de $17 \%$ de machos do crustáceo Hyalella azteca numa mistura de sete compostos farmacêuticos, entre eles o paracetamol (BORGMANN et al., 2007).

Tabela 9 - Concentração de paracetamol nos pontos analisados; nd significa que nada foi determinado.

\begin{tabular}{c|c|c|c|c|c|c}
\hline $\begin{array}{c}\text { Paracetamol } \\
\text { ng/L }\end{array}$ & Janeiro & Março & Abril & Junho & Setembro & Novembro \\
\hline CA/HO & nd & 6,5 & nd & nd & nd & nd \\
\hline HO/SU & nd & nd & nd & 7,3 & 12,4 & nd \\
\hline SU/NO & 143,2 & 75,1 & 95,3 & 284,5 & 172,1 & 305,1 \\
\hline NO/AM & 75,3 & 48,2 & 33,3 & 183,1 & 102,4 & 184,3 \\
\hline AM/FIM & 23,5 & 11,5 & 26,4 & 38,4 & 85,4 & 92,4 \\
\hline
\end{tabular}

O piroxicam (Tabela 10) apresentou a maior concentração em novembro no ponto SU/NO $\left(212,4 \mathrm{ng} / \mathrm{L}^{-1}\right)$ seguida por SU/NO $\left(174,2 \mathrm{ng} / \mathrm{L}^{-1}\right)$, SU/NO $\left(143,6 \mathrm{ng} / \mathrm{L}^{-1}\right)$, NO/AM $\left(142,4 \mathrm{ng} / \mathrm{L}^{-1}\right)$ e AM/FIM $\left(141,9 \mathrm{ng} / \mathrm{L}^{-1}\right)$.

Tabela 10 - Concentração de piroxican nos pontos analisados; nd significa que nada foi determinado.

\begin{tabular}{c|c|c|c|c|c|c}
\hline $\begin{array}{c}\text { Piroxican } \\
\text { ng/L }\end{array}$ & Janeiro & Março & Abril & Junho & Setembro & Novembro \\
\hline CA/HO & 5,6 & nd & nd & 8,4 & nd & 3,4 \\
\hline HO/SU & 6,5 & 8,2 & nd & 11,3 & 9,4 & 12,4 \\
\hline SU/NO & 143,6 & 98,4 & 75,4 & 98,4 & 174,2 & 212,4 \\
\hline NO/AM & 142,4 & 94,6 & 75,1 & 92,1 & 130,4 & 163,4 \\
\hline AM/FIM & 141,9 & 94,7 & 69,3 & 48,4 & 105,3 & 49,4 \\
\hline
\end{tabular}

Torre et al. (2014) menciona concentração de diclofenaco $\left(1200 \mathrm{ng} / \mathrm{L}^{-1}\right)$, naproxeno $\left(390 \mathrm{ng} / \mathrm{L}^{-1}\right)$, ibuprofeno $\left(3400 \mathrm{ng} / \mathrm{L}^{-1}\right)$, paracetamol $\left(10000 \mathrm{ng} / \mathrm{L}^{-1}\right)$, metoprolol $\left(2200 \mathrm{ng} / \mathrm{L}^{-1}\right)$ e propranalol $\left(590 \mathrm{ng} / \mathrm{L}^{-1}\right)$ detectadas em águas superficiais na qual foram observadas por Daughton e Ternes (1999); Boxal (2004). Américo et al. (2013) relata que na pesquisa de Vulliet et al. foram registradas concentrações 
em ng/ $\mathrm{L}^{-1}$ de analgésicos e anti-inflamatório (diclofenaco, ibuprofeno, naproxeno e paracetamol), do $\beta$-bloqueador atenolol, Camacho-Munoz et al. também reportaram a existência das mesmas classes de fármacos em águas superficiais e efluentes de ETE na Bacia Hidrográfica Donana Park (Espanha).

Petrovic et al. (2005) afirma que a eliminação de fármacos polares e ácidos como o diclofenaco e o ibuprofeno em ETEs merece atenção especial, visto que devido as suas propriedades físicoquímicas, como solubilidade em água e baixa degradabilidade, estes compostos podem atravessar todos os processos de filtração e atingir as águas subterrâneas e inclusive a água potável.

No Brasil ainda existem poucas informações sobre a ocorrência de compostos farmacológicos no meio ambiente e nas estações de tratamento de água e esgoto. Resíduos de fármacos como ácido clofíbrico, diclofenaco e naproxeno foram encontrados em rios e córregos do Estado do Rio de Janeiro, com maiores concentrações nas águas superficiais próximas aos centros urbanos com contaminações provenientes [de] águas residuárias municipais (Stumpf et al., 1999). No Reservatório Billings, Estado de São Paulo, fármacos utilizados como antipiréticos, betabloqueadores, analgésicos e anti-inflamatórios foram identificados, sendo que a presença destes compostos foram explicadas pelas condições sanitárias do corpo hídrico que recebe um volume considerável de efluentes sanitários sem tratamento provenientes das moradias no seu entorno, além de efluentes originários de diversas atividades de natureza desconhecida em sua bacia hidrográfica (ALMEIDA; WEBBER, 2005; AMÉRICO et al., 2013).

No Brasil, a principal fonte de contaminação ambiental das águas se dá através de esgotos domésticos não tratados, uma vez que a grande maioria dos efluentes urbanos não passa pelo processo de tratamento terciário para remoção de nutrientes e desinfecção. De acordo com a Pesquisa Nacional de Saneamento Básico (realizada pelo IBGE, em 2002) 82\% desses efluentes são descartados em rios que são utilizados para o abastecimento de água potável (SODRÉ et al., 2010; SILVA; COLLINS, 2011).

Amostras de água superficial dos rios Atibaia, Ribeirão Anhumas, Capivari e Jundiaí (Bacia do rio Atibaia), localizados na região metropolitana da cidade de Campinas, no Estado de São Paulo, em 2006, utilizando a técnica de SPE para extração dos analitos e a HPLC com detectores DAD e fluorescência, revelou a presença de paracetamol, cafeína, ácido acetilsalicílico, di- $n$-butilftalato, bisfenol $A$, $17 \beta$-estradiol e 17a-etinilestradiol nas faixas de concentração entre 0,005 até 41,7 $\mu \mathrm{g} / \mathrm{L}^{-1}$ refletindo, assim, o baixo percentual de tratamento da Região Metropolitana de Campinas, especialmente nas regiões onde a densidade populacional é mais elevada (SODRÉ et al. 2007; SILVA; COLLINS, 2011). 


\section{CONCLUSÕES FINAIS}

De um modo geral o ponto que mais apresentou fármacos foi SU/NO. O município de Sumaré trata aproximadamente $20 \%$ de seu esgoto, o restante é descartado in natura, é o município que menos trata esgoto, isto justifica as concentrações observadas após o munícipio. É possível observar uma biodegradação dos fármacos no decorrer do Ribeirão Quilombo.

Este estudo mostra que o Ribeirão Quilombo é castigado e consecutivamente leva este castigo para o Rio Piracicaba.

\section{REFERÊNCIAS}

ALDER, A. C. et al. Fate of beta-blocker human pharmaceuticals in surface water: comparison of measured and simulated concentrations in the Glatt Valley Watershed, Switzerland. Water Research, v. 44, n. 3, p. 936-948, 2010.

ALMEIDA, G. A.; WEBBER, R. R. Fármacos na represa Billings. Revista Saúde e Ambiente, v. 6, n. 2, p. 7-13, 2005.

AMÉRICO, J. H. P. et al. Fármacos em uma estação de tratamento de esgoto na região Centro-Oeste do Brasil e os riscos aos recursos hídricos. Revista Brasileira de Recursos Hídricos, v. 17, n. 2, p. 61-67, 2012.

BORGMANN, U. et al. Effect of a mixture of seven pharmaceuticals on Hyalella azteca over multiple generations. Chemosphere, v. 66, n. 7, p. 1278-1283, 2007.

BURKE, A.; SMYTH, E.; FITZGERAL, D. G. A. Analgesic-antipyretic agents; pharmacotherapy of gout. In: BRUNTON, L. L, (Coord.), Goodman \& Gilmans the pharmacological basis of therapeutics. 11th ed. New York: McGraw Hill, p. 671715, 2006.

CALAMARI, D. et al. Strategic Survey of Therapeutic Drugs in the Rivers Po and Lambro in Northern Italy. Environmental. Science Technology, v. 37, n. 7, p. 12411248, 2003.

CASTIGLIONI, S. et al. Removal of pharmaceuticals in sewage treatment plants in Italy. Environmental. Science Technology, v. 40, n. 1, p. 357-363, 2006.

CASTRO, T. F. et al. Identificação de diclofenaco de sódio em esgoto sanitário da ETE de São Carlos-SP. Disponível em: <https://uspdigital.usp.br/siicusp/ cdOnlineTrabalhoVisualizarResumo?numerolnscricaoTrabalho=2223\&numeroEdicao =19>. Acesso em: 26 dez. 2014. 
DORNE, J. L. C. M..; RAGAS, A. M. J.; FRAMPTOM, G. K.; SPURGEON, D. S.; LEWIS, D. F. Trends in human risk assessment of pharmaceuticals. Analytical and Bioanalytical Chemistry, v. 387, n. 4, p. 1167-1172, 2007.

EL-OBEID, H. A.; AL-BADR, A. A. Analytical of Drugs Substances 14. Florey, K.; San Diego: ed Academic Press, 1985.

FENT, K.; WESTON, A. A.; CAMINADA, D. Ecotoxicology of human pharmaceuticals. Aquatic Toxicology, v. 76, n. 2, p. 122-159, 2006.

FOWLER, P. D. et al. Plasma and synovial fluid concentrations of diclofenac sodium and its major hydroxylated metabolites during long-term treatment of rheumatoid arthritis. European Journal of Clinical Pharmacology, v. 25, n. 3, p. 389-394,1983.

GELLER, M.; KRYMCHANTOWSKI, A. V.; STEINBRUCH, M.; CUNHA, K. S.; RIBEIRO, M. G.; OLIVEIRA, L.; OZERI, D.; DAHER, J. P. L. Utilização do diclofenaco na prática clínica: revisão das evidências terapêuticas e ações farmacológicas. Revista Brasileira de Clinica Medica, v. 10, n. 1, p. 29-38, 2012.

GOTTIEB, N. L. The art and science of nonsteroidal anti-inflammatory drug selection. Seminars in Arthritis and Rheumatism, v. 15, n. 2S1, p.1-3, 1985.

HECKMANN, L. H. et al. Chronic toxicity of ibuprofen to Daphnia magna: Effects on life history traits and population dynamics. Toxicology Letters, v. 172, n.3, p.137145, 2007.

HERNANDO, M. D. et al. LC-MS analysis of basic pharmaceuticals (beta-blockers and anti-ulcer agents) in wastewater and surface water. Analytical Chemistry, v. 26, n. 6, p.581-594, 2007.

HIGGS, J. D. et al. Analyticlorey, K. ed. San Diego: Academic Press, 1999, p. 282.

HONG, H. N. et al. Analysis of the effects diclofenac has on Japanese medaka (Oryzias latipes) using real-time PCR. Chemosphere, Oxford, v. 67, n. 11, p. 21152121, 2007.

HUGGET, D. B. et al. Toxicity of select beta adrenergic receptor-blocking pharmaceuticals (B-blockers) on aquatic organisms. Archives of Environmental Contamination and Toxicology, v. 43, n. 2, p. 229-235, 2002.

ISIDORI, M. et al. Ecotoxicity of naproxen and its phototransformation products. Science of the Total Environment, Amsterdam, v. 348, n. 1-3, p. 93-101, 2005.

$\mathrm{KIM}, \mathrm{Y}$. et al. Aquatic toxicity of acetaminophen, carbamazepine, cimetidine, diltiazem and six major sulfonamides, and their potential ecological risks in Korea. Environment International, v. 33, n. 3, p. 370-37, 2007.

LIAUW, $\mathrm{H}$. et al. Effects of diclofenac on synovial eicosanoid product formation in arthritic patients. Journal of Clinical Pharmacology, v. 25, p. 455-74, 1985. 
MOSQUERA, V. et al. Thermodynamics of micellization of surfactants of low aggregation number: the aggregation of propranolol hydrochloride. Journal Colloid Interface Science, v. 210, n. 1, p. 97-102, 1999.

NOLDER, K. et al. Development of a multi-residue analytical method, based on liquid chromatography-tandem mass spectrometry, for the simultaneous determination of 46 micro-contaminants in aqueous samples. Journal of chromatography, v. 1217, n. 42, p. 6511-6521, 2010.

PASQUINI, N, C. Diagnóstico da qualidade da água do Ribeirão Quilombo, Americana, Estado de São Paulo. Educação Ambiental em Ação, v. 49, 2014. Disponível em: <http://www.revistaea.org/search.php?resultado= 1\&palavra=pasquini\&exemplar=all\&cadastrese=Procurar $>$. Acesso em: 22 dez. 2014a.

Uso potencial como fonte de energia de lodo proveniente de esgoto doméstico e industrial. Revista Ciências Extas e Naturais, v. 16, n. 1, p. 35-57, $2014 b$.

. Educação ambiental no controle de aedes aegypti, uma estratégia de combate e prevenção. Educação Ambiental em Ação, v. 41, 2012. Disponível em: http://www.revistaea.org/search.php?resultado=1\&palavra=pasquini\&exemplar=all\&c adastrese=Procurar. Acesso em: 22 dez. 2014.

PETROVIC, M. et al. Liquid chromatography-tandem mass spectrometry for the analysis of pharmaceutical residues in environmental samples: a review. Journal of Chromatography A, v. 1067, n. 1-2, p.1-14, 2005.

POMATI, F. et al. Effects of erythromycin, tetracycline and ibuprofen on the growth of Synechocystis sp and Lemna minor. Aquatic Toxicology, v. 67, n. 4, p. 387-396, 2004

PDMBRQ. Plano Diretor de Macrodrenagem da Bacia do Ribeirão Quilombo. Disponível em: <http://www.comitepcj.sp.gov.br/download/Plano-MacroDrenQuilombo-Anexos_Jan-02.pdf>. Acesso em: 10 mar. 2015.

RICHARDS, S. M. et al. Effects of pharmaceutical mixtures in aquatic microcosms. Environmental Toxicology and Chemistry, v. 23, n. 4, p. 1035-1042, 2004.

SCHWAIGER, J. et al. Toxic effects of the non-steroidal anti-inflammatory drug diclofenac, Part I: histopathological alterations and bioaccumulation in rainbow trout. Aquatic Toxicology, Amsterdam, v.68, n.2, p.141-150, 2004.

SILVA, C. G. A.; COLLINS, C. H. Aplicações de cromatografia líquida de alta eficiência para o estudo de poluentes orgânicos emergentes. Química Nova, v. 34, n. 4, p. 665-676, 2011.

SODRÉ, F. F.; LOCATELLI, M. A. F.; JARDIM, W. F. Occurence of emerging contaminants in Brazilian drinking waters: A sewage-to-tap issue. Water, Air, Soil Pollut, v. 206, n. 1-4, p. 57-67, 2010. 
; MONTAGNER, M. A. F.; JARDIM, W. F. Identificação e quantificação de interferentes endócrinos em água potável na cidade de Campinas-SP. XXX RASBQ, Águas de Lindóia, 2007.

STUMPF, M.; TERNES, T. A.; WILKEN, R. D.; RODRIGUES, S. V.; BAUMANN, W. Polar drug residues in sewage and natural waters in the state of Rio de Janeiro,Brazil. The Science of Total Environmmental, v. 225, n. 1-2, p.135$141,1999$.

TORRE, J. A. C. et al. Presença de fármacos em águas residuais e eficácia de processos convencionais de eliminação, 2014. Disponível em:

<http://www.ciccp.es/biblio_digital//citema_III/congreso/pdf/010511.pdf>. Acesso em: 16 dez. 2014.

TUNDISI, J. G. Água no século XXI: enfrentando a escassez. 2.ed. São Carlos: Rima, 2003, 248p.

VAGA, R. et al. Determination of antihypertensive and anti-ullcer agents from surface water with solid-phase extraction-liquid chromatography-electrospray ionization tandem mass spectrometry. Talanta, v. 83, n. 5, p.1447-14450, 2011.

VAZQUEZ-ROIG, P. et al. Determination of pharmaceuticals in soils and sediments by pressurized liquid extraction and liquid chromatography tandem mass spectrometry. Journal Chromatography, v. 1217, p. 2471-2483, 2010.

WEIGEL, S.; KALLENBORN, R.; HUHNERFUSS, H. Simultaneous solid-phase extraction of acidic, neutral and basic pharmaceuticals from aqueous samples at ambient (neutral) $\mathrm{pH}$ and their determination by das chromatography-mass spectrometry. Journal of Chromatography A, v. 1023, n. 183-195, 2004.

Artigo recebido em: 01/03/2015

Artigo aprovado em: 14/06/2016 